\title{
Influence of basin geometry on equilibrium and stability of double inlet systems
}

\author{
R.L. Brouwer ${ }^{1}$, H.M. Schuttelaars ${ }^{2}$ and P.C. Roos $^{3}$ \\ ${ }^{1}$ Section of Hydraulic Engineering, Delft University of Technology, P.O. Box 5048, 2600 GA Delft, The Netherlands, \\ r.1.brouwer@tudelft.nl \\ ${ }^{2}$ Institute of Applied Mathematics, Delft University of Technology, Mekelweg 4, 2600 GA Delft, The Netherlands, \\ h.m.schuttelaars@tudelft.nl \\ ${ }^{3}$ Department of Water Engineering and Management, University of Twente, P.O. Box 217, 7500 AE, Enschede, The Netherlands, \\ p.c.roos@utwente.nl
}

\begin{abstract}
This study investigates the influence of basin geometry on the cross-sectional stability of double inlet systems. The inlet is in equilibrium when the amplitude of the inlet velocities equals the equilibrium velocity $\left(\sim 1 \mathrm{~m} \mathrm{~s}^{-1}\right)$. This equilibrium is stable when after a perturbation the cross-sections of both inlets return to their original equilibrium value. The necessary amplitudes of the inlet velocities are obtained using an idealized 2DH hydrodynamic that calculates tidal elevation and flow in a geometry consisting of several adjacent rectangular compartments.

Model results suggest that regardless of the inclusion or exclusion of bottom friction in the basin, stable equilibrium states exist. Qualitatively, the influence of basin geometry does not change the presence of stable equilibrium. Quantitatively, however, taking a basin surface area of $1200 \mathrm{~km}^{2}$, equilibrium values can differ up to a factor 2 depending on the geometry of the basin.
\end{abstract}

\section{INTRODUCTION}

Barrier island coasts are highly dynamical systems that serve as a first defense for the hinter lying mainland. Examples are the Wadden Sea coast of the Netherlands, Germany and Denmark, the U.S. East Coast and the Ria Formosa in Southern Portugal. Understanding the mechanisms causing these (multiple) tidal inlet systems to be cross-sectionally stable is of importance to anticipate the effects of natural or man-made changes in these systems. Examples are sea level rise, barrier island breaching and basin reduction. Following Escoffier [1940] an inlet is considered to be in equilibrium when the amplitude of the inlet velocity equals the equilibrium velocity. The equilibrium is stable when after a perturbation the cross-section of that inlet returns to the original equilibrium state.

In calculating the amplitude of the inlet velocity it is customary to use a semi-empirical cross-sectionally averaged equation for the flow in the inlet and to assume a uniformly fluctuating water level, the so-called pumping mode, for the basin. In particular the use of the pumping mode in these lumped models needs justification as by definition water levels inside the basin vary in amplitude as well as phase. It is postulated that the validity of the use of the pumping mode depends among others on the basin dimensions including depth and geometry, i.e. length to width ratio when the surface area is assumed constant. This in turn can cause inlet velocities to vary and therefore change the stability of the inlet system.

The goal of this study is twofold: 1) to investigate the influence of basin geometry on the equilibrium and stability of double inlet systems and 2) to compare the results with a cross-sectionally averaged pumping mode model (e.g. van de Kreeke et al., 2008). To this end, an idealized 2DH hydrodynamic model is developed based on Roos \& Schuttelaars [2011] and Roos et al. [2011] that calculates tidal elevation and flow in a schematized geometry of a tidal inlet system. This approach will be explained in the next section together with the definition of cross-sectional stability. Subsequently, the model results are presented using so-called flow diagrams. We finalize our study with a discussion, conclusions and an outlook for future research.

\section{METHODOLOGY}

\section{Cross-sectional stability}

In this study the focus is on cross-sectional stability. Following Escoffier [1940] an inlet is assumed to be in equilibrium if the amplitude of the cross-sectionally averaged inlet velocity is equal to the so-called equilibrium velocity $\hat{u}_{\text {eq }}$, generally taken as $1 \mathrm{~m} \mathrm{~s}^{-1}$ [Bruun et al., 1978]. The equilibrium is stable when after a perturbation of the equilibrium, the cross-sectional areas return to these equilibrium values. For inlets that are in equilibrium and assuming average weather conditions (as opposed to storm conditions) there is a balance between the volume of sediment entering and leaving the inlet. Following van de Kreeke [2004], the volume of sediment entering the inlet is taken as a constant fraction of the littoral drift, while the volume leaving the inlet is taken proportional to a power of the ebb tidal velocity amplitude. The difference between the amount of sediment that enters and leaves the inlet during a tidal cycle is uniformly distributed over the inlet length if this difference is positive; if negative the inlet is eroded uniformly. Hence, sediment exchange between inlet and basin is assumed to be negligible. Under these assumptions, the rate of change of the cross-sectional area can be written as [van de Kreeke et al., 2008; de Swart \& Zimmerman, 2009] 


$$
\frac{d A_{j}}{d t}=\frac{M}{l_{j}}\left(\left(\frac{\hat{u}_{j}}{\hat{u}_{e q}}\right)^{n}-1\right) .
$$

Here, $A_{j}$ is the cross-sectional area of inlet $j\left(\mathrm{~m}^{2}\right) ; t$ is time $(\mathrm{s}) ; l_{j}$ is the length of inlet $j(\mathrm{~m}) ; M$ is a constant fraction of the littoral drift $\left(\mathrm{m}^{3} \mathrm{~s}^{-1}\right) ; \hat{u}_{j}$ is the cross-sectionally averaged velocity amplitude of compartment $j\left(\mathrm{~m} \mathrm{~s}^{-1}\right)$; and $n$ is a power whose value depends on the adopted sand-transport law. Here $n$ is assumed to be 3. If $\hat{u}_{j}=\hat{u}_{e q}$, it follows that $d A / d t=0$. This implies that the inlet system is in equilibrium.

\section{Hydrodynamic model formulation}

As shown in Eq. (1), the response of the inlet cross-sectional area and the equilibrium state is governed by the amplitude of the cross-sectionally averaged inlet velocities $\hat{u}_{j}$ that, in general, follows from a numerical or analytical model. In this study, the velocities are calculated using an idealized $2 \mathrm{DH}$ hydrodynamic model based on the modeling approach described in Roos \& Schuttelaars [2011]; Roos et al. [2011]. Compared to the more classical lumped models of tidal inlets [e.g. van de Kreeke et al., 2008], our new hydrodynamic model has the following properties. (1) Since the adjacent sea/ocean is contained in the model

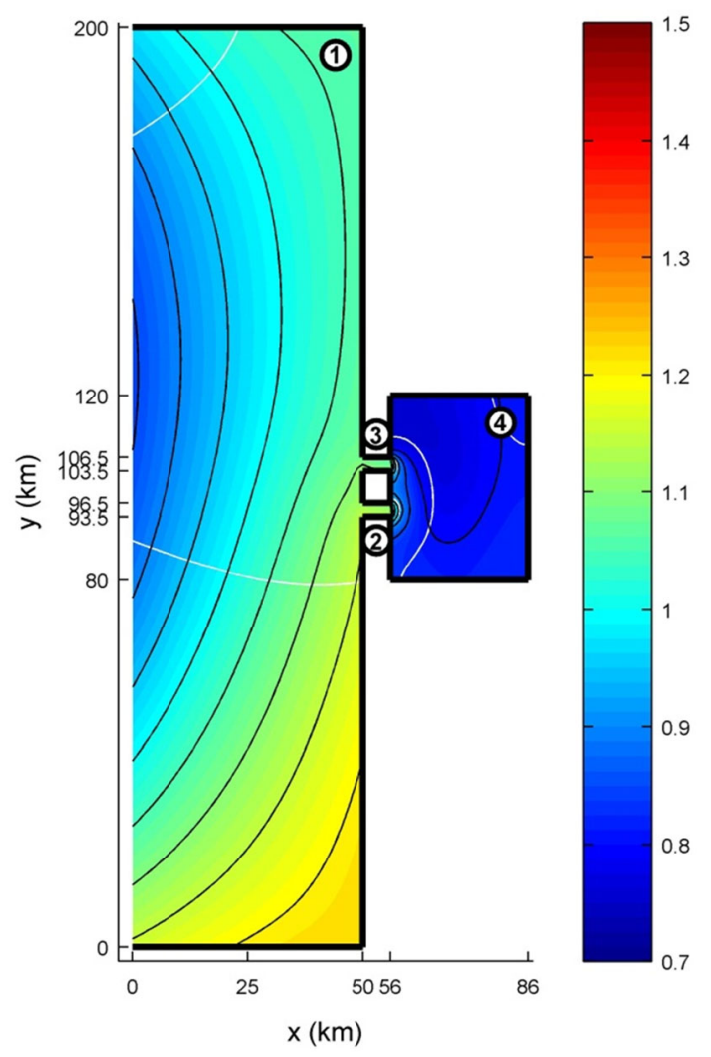

Figure 1. Co-tidal chart for a double inlet system with a basin geometry of $30 \times 40 \mathrm{~km}$. Elevation amplitudes are in meters, cophase lines are depicted in white with intervals of $30^{\circ}$ and corange contours are depicted in black with intervals of $0.05 \mathrm{~m}$. Compartment dimensions are listed in Table 1.
Table 1. Compartment dimensions of an inlet system representing the Marsdiep-Vlie sysitem in the western Dutch Wadden Sea.

\begin{tabular}{ccccc}
\hline Fig. & $\boldsymbol{j}$ & $\boldsymbol{b}_{\boldsymbol{j}}(\mathbf{k m})$ & $\boldsymbol{l}_{\boldsymbol{j}}(\mathbf{k m})$ & $\boldsymbol{h}_{\boldsymbol{j}}(\mathbf{m})$ \\
\hline 1,3 & 1 & 200 & 50 & 25 \\
1,3 & 2,3 & 3 & 6 & 15 \\
1,3 & 4 & 40 & 30 & $5^{(1)} ; 1 \times 10^{6(3)}$ \\
$2 \mathrm{a}, \mathrm{b}, \mathrm{c}$ & 1 & 200 & 50 & 25 \\
$2 \mathrm{a}, \mathrm{b}, \mathrm{c}$ & 2,3 & variable & 6 & variable \\
$2 \mathrm{a}, \mathrm{b}, \mathrm{c}$ & 4 & $80^{(\mathrm{a})} ; 40^{(\mathrm{b})} ; 20^{(\mathrm{c})}$ & $15^{(\mathrm{a})} ; 30^{(\mathrm{b})} ; 60^{(\mathrm{c})}$ & 5 \\
4 & 1 & 200 & 50 & 25 \\
4 & 2,3 & variable & 6 & variable \\
4 & 4 & 40 & 30 & $1 \times 10^{6}$ \\
\hline
\end{tabular}

Superscripts above a parameter value refer to the corresponding figure

geometry, the tidal wave past the inlet system is part of the solution. In turn, this implies that the amplitude and phase differences between the two inlets are automatically calculated and need not be imposed externally. (2) Amplitude and phase differences within the tidal basin are accounted for, which is important for rather elongated and shallow tidal basins. (3) Bottom friction in the basin is also accounted for, which is particularly realistic for shallow tidal basins. (4) The hydrodynamic method is quick, thus allowing for extensive sensitivity analyses with respect to the geometrical and physical characteristics of the system. (5) Our schematization ignores the complex channel-shoal patterns by assuming a uniform depth. (6) The model can be readily extended to systems with more than two inlets.

Our model calculates tidal elevation and flow in a geometry consisting of several adjacent rectangular compartments. Figure 1 shows an example of such a geometry for a double inlet system resembling the Marsdiep-Vlie system in the Dutch Wadden Sea. It consists of four compartments of length $l_{j}$, width $b_{j}$ and (uniform) depth $h_{j}(j=1, \ldots, 4)$. Compartment 1 , which has an open boundary to the left, represents the ocean/sea. Compartment 2 and 3 are the two inlet channels of rectangular cross-section. Compartment 4 is the tidal basin. In each compartment, conservation of momentum and mass is expressed by the depth-averaged shallow water equations including Coriolis effects, and linear bottom friction on the $f$-plane:

$$
\begin{aligned}
& \frac{\partial u_{j}}{\partial t}-f v_{j}+\frac{r_{j} u_{j}}{h_{j}}=-g \frac{\partial \eta_{j}}{\partial x} \\
& \frac{\partial v_{j}}{\partial t}+f u_{j}+\frac{r_{j} v_{j}}{h_{j}}=-g \frac{\partial \eta_{j}}{\partial y} \\
& \frac{\partial \eta_{j}}{\partial t}+h_{j}\left[\frac{\partial u_{j}}{\partial x}+\frac{\partial v_{j}}{\partial y}\right]=0 .
\end{aligned}
$$


Table 2. General parameter values resembling the Marsdiep-Vlie system in the western Dutch Wadden Sea.

\begin{tabular}{ccccccccc}
\hline $\boldsymbol{M}\left(\boldsymbol{m}^{3} \mathbf{y e a r ^ { - 1 }}\right)$ & $\hat{\boldsymbol{u}}_{\boldsymbol{e q}}\left(\boldsymbol{m} \boldsymbol{s}^{-1}\right)$ & $\boldsymbol{\Omega}\left(\mathrm{rad} \mathrm{s}^{-1}\right)$ & $\boldsymbol{g}\left({ }^{\boldsymbol{o}} \boldsymbol{N}\right)$ & $\boldsymbol{c}_{\boldsymbol{D}}(-)$ & $\gamma(-)$ & $\boldsymbol{Z}_{M 2}(\boldsymbol{m})$ & $\boldsymbol{\omega}\left(\boldsymbol{s}^{-1}\right)$ & $\boldsymbol{g}\left(\boldsymbol{m} \boldsymbol{s}^{-2}\right)$ \\
\hline $5 \times 10^{5}$ & 1 & $7.292 \times 10^{-5}$ & 53 & $2.5 \times 10^{-3}$ & 0.005 & 1 & $1.4 \times 10^{-4}$ & 9.81 \\
\hline
\end{tabular}

For compartment $j, u_{j}$ and $v_{j}$ are the depth-averaged flow velocity components in along-basin (x)- and cross-basin (y)-direction, respectively, and $\eta_{j}$ is the free surface elevation. Furthermore, $f=$ $2 \Omega \sin \vartheta$ is a Coriolis parameter (with $\Omega=7.292 \times 10^{-5} \mathrm{rad} \mathrm{s}^{-1}$ the angular frequency of the Earth's rotation and $\vartheta \sim 53^{\circ} \mathrm{N}$ the central latitude of the system) and $g=9.81 \mathrm{~m} \mathrm{~s}^{-2}$ the gravitational acceleration. The linear bottom friction coefficient is defined as $r_{j}$ $=8 c_{D} U_{j} / 3 \pi$ obtained from Lorentz' linearization of a quadratic friction law [Zimmerman, 1982] with a default value of the drag coefficient $c_{D}=2.5 \times 10^{-3}$. The current amplitude of a classical Kelvin wave without bottom friction is assumed as the typical flow velocity scale $U_{j}=Z_{M 2} \sqrt{ }\left(g / h_{j}\right)$. Here $Z_{M 2}=1 \mathrm{~m}$ is typical for the dominant M2-tide.

The model geometry displays different types of boundaries. At the closed boundaries, a no-normal flow condition is imposed. Next, continuity of elevation and normal flux is required across the topographic steps between the adjacent compartments.

Analogous to the classical Taylor [1922] problem, the system is forced by a single incoming Kelvin wave with angular frequency $\omega$ and typical elevation amplitude $Z_{M 2}$, entering through the open boundary of compartment 1 . Due to the Coriolis effect the Kelvin wave travels upward along the coast past the two inlets, thus forcing the flow in the inlet system. This effect is negligible inside the inlets, as its dimensions are generally small compared to the Rossby deformation radius. The traveling Kelvin wave along with other waves is allowed to radiate outward at the open boundary of compartment 1 .

\section{Flow diagram}

To determine the equilibrium cross-sectional areas and their stability, the results are expressed in terms of a so-called flow diagram [van de Kreeke et al., 2008]. Using the hydrodynamic model described above the cross-sectionally averaged velocity amplitudes $\hat{u}_{1}$ and $\hat{u}_{2}$ are calculated for multiple combinations of $\left(A_{1}, A_{2}\right)$. The cross-sections are varied by enlarging the width $b_{j}$ of the inlet compartments and calculating the corresponding depth $h_{j}$. This is done using the assumption of a geometrically similar rectangular cross-section [O'Brien \& Dean, 1972]. Hence, the ratio $\gamma=h_{j} / b_{j}$ is constant for all cross-sections; where $\gamma$ is chosen to be 0.005 . From $\hat{u}_{1}\left(A_{1}, A_{2}\right)$ and $\hat{u}_{2}\left(A_{1}, A_{2}\right)$, equilibrium velocity curves are constructed for both inlets. The equilibrium velocity curves represent the locus of $\left(A_{1}, A_{2}\right)$-values for which $\hat{u}_{1}=\hat{u}_{e q}$ and $\hat{u}_{2}=\hat{u}_{e q}$, respectively. The intersections of the two curves represent sets of equilibrium cross-sectional areas. To determine the stability of the equilibrium, vectors are added to the flow diagram. These vectors are the unit vectors in the direction of $d \vec{A} / d t$ calculated from Eq. (1). The unit vectors indicate the direction in which the values of cross-sectional areas change when they are not in equilibrium.

\section{RESULTS}

In this study, three basin geometries $\left(l_{4} \times b_{4}\right)$ are chosen with a constant basin surface of $1200 \mathrm{~km}^{2}: 15 \times 80 \mathrm{~km}, 30 \times 40 \mathrm{~km}$ and $60 \times 20 \mathrm{~km}$ (see Table 1). Other general parameter values used in the calculations are denoted in Table 2 and are roughly based on a)

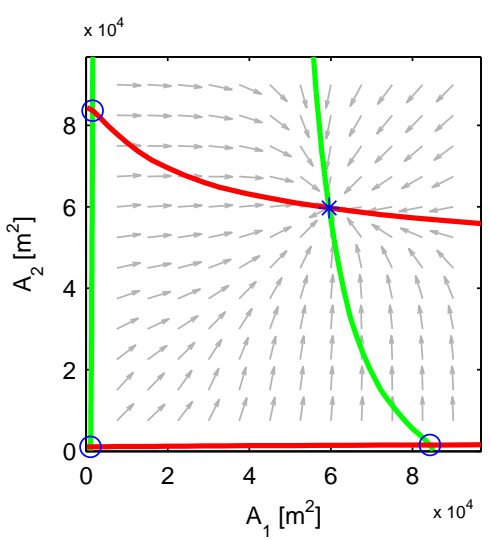

b)

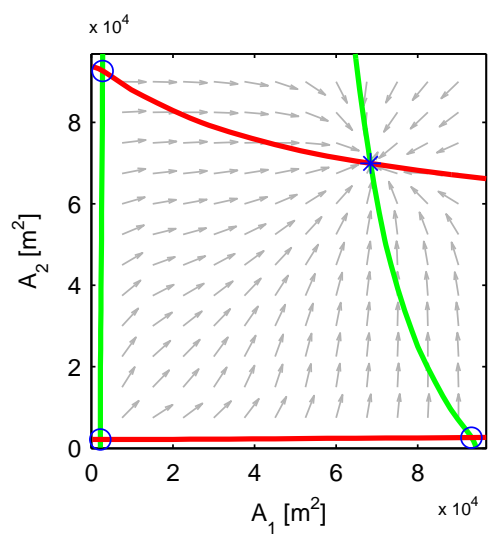

c)

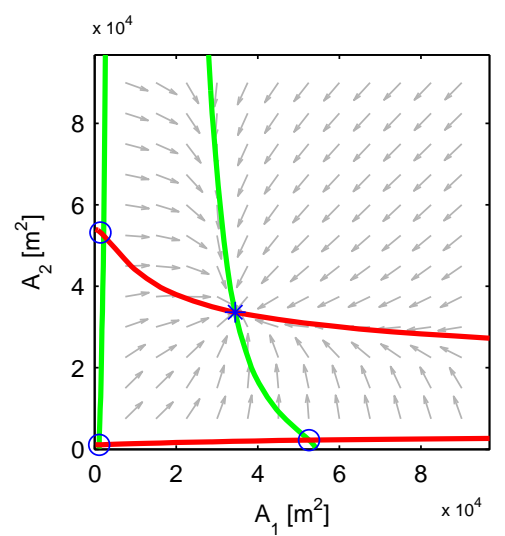

Figure 2. Flow diagrams for different basin geometries: a) $15 \times 80 \mathrm{~km}$, b) $30 \times 40 \mathrm{~km}$ and c) $60 \times 20 \mathrm{~km}$. The green and red solid line corresponds to the equilibrium velocity curves of inlet 1 and 2, respectively. The gray vectors indicate the direction in which the values of the cross-sectional areas change when they are not in equilibrium. Blue circles indicate an unstable equilibrium and the blue cross a stable one. 
the Marsdiep-Vlie system in the western Dutch Wadden Sea. For each configuration the centerline of the tidal basin is on the same position as the centerline of the ocean and the centerlines of the inlet channels are $10 \mathrm{~km}$ apart and symmetrically positioned with respect to the centerlines of the ocean and basin.

\section{Bottom friction in the basin}

A typical result of the model with dimensions resembling the Marsdiep-Vlie system is shown in Fig. 1, which shows a co-tidal chart for a basin geometry of $30 \times 40 \mathrm{~km}$. Other dimensions of the system are listed in Table 1. It follows that due to bottom friction amplitudes and phases show a spatial variability of approximately $10 \mathrm{~cm}$ and $30^{\circ}$, respectively. Moreover, amplitudes decay from approximately $1.1 \mathrm{~m}$ in the inlets to approximately $0.75 \mathrm{~m}$ inside the basin.

Flow diagrams are used to determine how this spatial variability of amplitudes and phases influences the stability of the system for different basin geometries. This is shown in Figs. $2 \mathrm{a}, \mathrm{b}$ and $\mathrm{c}$ for a basin geometry of $15 \times 80 \mathrm{~km}, 30 \times 40 \mathrm{~km}$ and $60 \times 20$ $\mathrm{km}$, respectively. It follows that for all three cases next to three unstable equilibriums, a single stable equilibrium exists. Moreover, the influence of basin geometry has a large quantitative influence on the equilibrium values of the cross-sectional areas. The largest equilibrium values are found for the $30 \times 40 \mathrm{~km}$

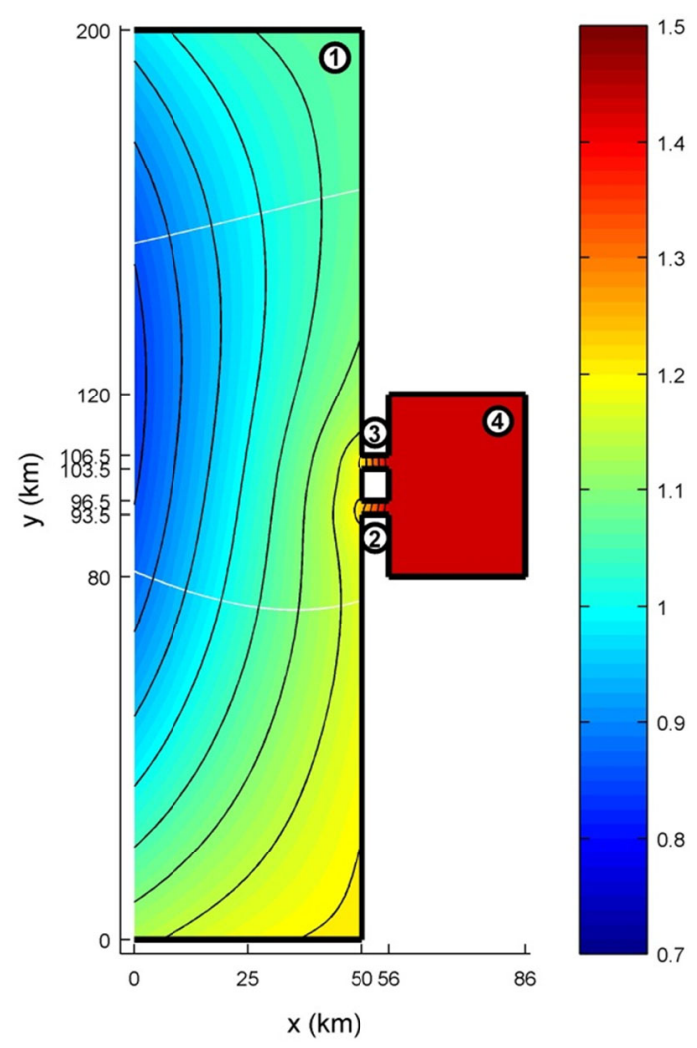

Figure 3. Co-tidal chart for a double inlet system with a basin geometry of $30 \times 40 \mathrm{~km}$. Elevation amplitudes are in meters, cophase lines are depicted in white with intervals of $30^{\circ}$ and corange contours are depicted in black with intervals of $0.05 \mathrm{~m}$. Compartment dimensions are listed in Table 1.

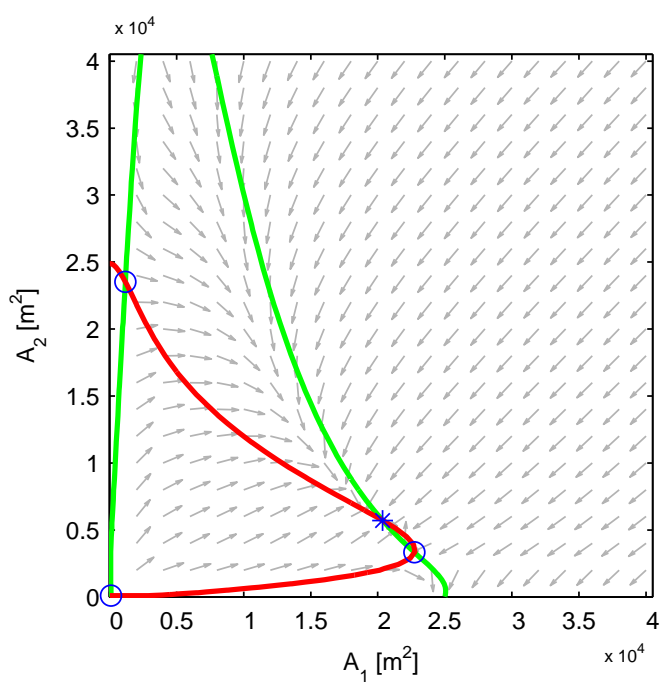

Figure 4. Flow diagram for a basin geometry of $30 \times 40 \mathrm{~km}$, no bottom friction in the basin $\left(\mathrm{c}_{\mathrm{D}}=10\right)$ and basin depth $h_{4}=1 \times 10^{6}$ $m$. The green and red solid line corresponds to the equilibrium velocity curves of inlet 1 and 2, respectively. The gray vectors indicate the direction in which the values of the cross-sectional areas change when they are not in equilibrium. Blue circles indicate an unstable equilibrium and the blue cross a stable one.

geometry (Fig. 2a), i.e. $\left(\mathrm{A}_{1}, \mathrm{~A}_{2}\right) \approx\left(7 \times 10^{4}, 7 \times 10^{4}\right)$. The other basin shapes lead to smaller equilibrium values (Figs. 2a and $2 \mathrm{c}$ ); $\left(\mathrm{A}_{1}, \mathrm{~A}_{2}\right) \approx\left(6 \times 10^{4}, 6 \times 10^{4}\right)$ for the $15 \times 80 \mathrm{~km}$ geometry and $\left(\mathrm{A}_{1}, \mathrm{~A}_{2}\right)$ $\approx\left(3.5 \times 10^{4}, 3.5 \times 10^{4}\right)$ for the $60 \times 20 \mathrm{~km}$ geometry. This leads to a difference in equilibrium values up to a factor 2 depending on the geometry of the basin.

\section{No bottom friction in the basin}

It is interesting to determine to what extent the results of our idealized 2DH hydrodynamic model can be compared with results from the classical lumped model approach. Apart from other assumptions, an important one in that approach is a uniformly fluctuating water level inside the basin. Results by van de Kreeke et al. [2008] show that for a double inlet system with a single basin and relatively long inlet channels two equilibriums exist, none of which is stable.

To approximate the pumping mode with our model we consider a basin geometry of $30 \times 40 \mathrm{~km}$ with $c_{D}=0$ and $h_{4}=1 \times 10^{6} \mathrm{~m}$. This results in the co-tidal chart depicted in Fig. 3. A clear difference with the case including bottom friiction (Fig. 1) is that in the absence of bottom friction amplitudes are amplified from approximately $1.25 \mathrm{~m}$ in the inlet channel to approximately $1.4 \mathrm{~m}$ inside the basin. Furthermore, amplitudes and phases in the basin do not display (visual) spatial variability. The corresponding flow diagram for the $30 \times 40 \mathrm{~km}$ basin is depicted in Fig. 4. It follows that, similar to the case with bottom friction, four equilibriums exist, one of which is stable with equilibrium values of $\left(\mathrm{A}_{1}, \mathrm{~A}_{2}\right) \approx$ $\left(2 \times 10^{4}, 0.6 \times 10^{4}\right)$. 


\section{DISCUSSION}

Care should be taken to compare the results of our model with the lumped model of van de Kreeke et al. [2008] as even with $c_{D}=$ 0 and $h_{4}=1 \times 10^{6} \mathrm{~m}$ the water motion does not satisfy the pumping mode approximation. Other differences are the presence of physical mechanisms automatically accounted for in our $2 \mathrm{DH}$ model approach. Examples are resonance, radiation damping and entrance/exit losses. Comparing Fig. 4 with Fig. $2 \mathrm{~b}$ shows that the equilibrium velocity curve of inlet 2 (red) has retreated to almost within the equilibrium velocity curve of inlet 1 (green). It is to be expected that when our model more closely approximates the assumptions in the model by van de Kreeke et al. [2008] the stable equilibrium at $\left(\mathrm{A}_{1}, \mathrm{~A}_{2}\right) \approx\left(2 \times 10^{4}, 0.6 \times 10^{4}\right)$ will disappear, thus leading to two unstable equilibriums.

\section{CONCLUSIONS AND OUTLOOK}

In this study, we have investigated the influence of basin geometry on the stability of double inlet systems. To this end, an idealized 2DH hydrodynamic model was developed that calculates the spatial characteristics of tidal flow in a schematized geometry of a tidal inlet. Tidal inlet stability has been investigated by combining the inlet velocity amplitudes from this model with Escoffier's [1940] classical stability method.

The flow diagrams based on our model suggest that regardless the inclusion or exclusion of bottom friction in the basin stable equilibrium states exist. Moreover, qualitatively the basin shape does not change the presence of stable equilibriums. Quantitatively, a more elongated basin shape, in $(x)$ - or $(y)$ direction, generally corresponds to significantly smaller equilibrium values. Specifically, taking a basin surface area of $1200 \mathrm{~km}^{2}$, equilibrium values can differ up to a factor of approximately 2 depending on basin shape.

Inspired by the above conclusions, future research should focus on the following aspects.

- Examine the cause(s) of the large influence of basin geometry on the equilibrium values when assuming a constant basin surface area.

- Extend the sensitivity analysis to investigate the roles of physical mechanisms such as bottom friction, radiation damping, resonance and entrance/exit losses on the stability of double inlet systems.
- Investigate the influence of the position of the inlet channels with respect to where they connect the ocean to the tidal basin (in our case this position was assumed constant and the mutual distance between the inlet channel was relatively short).

- Investigate the consequences of alternatives for the similarity approach regarding the cross-sections of the tidal inlets (by which we assumed a constant factor $\gamma$ when constructing the flow diagrams in Figs. 2 and 4).

\section{REFERENCES}

Bruun, P. Mehta, A.P., Johnsson, I.G. (1978), Stability of tidal inlets: theory and engineering, Elsevier Scientific Publishing Co., The Netherlands.

Escoffier, F.F. (1940), The stability of tidal inlets, Shore and Beach, 8 (4), 111-114.

van de Kreeke, J. (2004), Equilibrium and cross-sectional stability of tidal inlets: application to the Frisian Inlet before and after basin reduction, Coastal Engineering, 51 (5-6), 337-350, doi: 10.1016/j.coastaleng.2004.05.002.

van de Kreeke, J., Brouwer, R.L., Zitman, T.J., Schuttelaars, H.M. (2008), The effect of a topographic high on the morphological stability of a two-inlet bay system, Coastal Engineering, 55 (4), 319-332, doi:10.1016/j.coastaleng.2007.11.010.

O'Brien, M.P. and Dean, R.G. (1972), Hydraulics and sedimentary stability of coastal inlets, $13^{\text {th }}$ International Conference on Coastal Engineering, Vancouver, Canada, 761-780.

Roos, P.C. and Schuttelaars, H.M. (2011), Influence of topography on tide propagation and amplification in semienclosed basins, Ocean Dynamics, 61 (1), 21-38, doi:10.1016/S0278-4343(97)00007-1.

Roos, P.C., Velema, J.J., Hulscher, S.J.M.H., Stolk, A. (2011), An idealized model of tidal dynamics in the North Sea: resonance properties and response to large-scale changes, Ocean Dynamics, 61 (12), 2019-2035, doi:10.1007/s10236-011-0456-x

de Swart, H.E., Zimmerman, J.T.F. (2009), Morphodynamics of Tidal Inlet Systems, Annual Review of Fluid Mechanics, 41, 203-229, doi:10.1146/annurev.fluid.010908.165159.

Taylor, G.I. (1922), Tidal oscillations in gulfs and rectangular basins, Proceedings of the London Mathematical Society, s2-20 (1), 148-181.

Zimmerman, J.T.F. (1982), On the Lorentz linearization of a quadratically damped forced oscillator, Physics Letters, 89A, 123-124. 